\title{
Phase I clinical trial of a novel peptide vaccine in combination with UFT/LV for metastatic colorectal cancer
}

\author{
KIYOTAKA OKUNO $^{1}$, FUMIAKI SUGIURA ${ }^{1}$, JIN-ICHI HIDA ${ }^{1}$, TADAO TOKORO ${ }^{1}$, \\ EIZABURO ISHIMARU $^{1}$, YASUSHI SUKEGAWA ${ }^{2}$ and KAZUKI UEDA ${ }^{1}$ \\ ${ }^{1}$ Department of Surgery, and ${ }^{2}$ Institute of Immunotherapy for Cancer, \\ Kinki University Faculty of Medicine, Osaka-sayama 589-8511, Japan
}

Received October 8, 2010; Accepted November 30, 2010

DOI: $10.3892 / \mathrm{etm} .2010 .182$

\begin{abstract}
To test the safety and immune responses of a novel peptide vaccine derived from RNF43 (ring finger protein 43) and TOMM34 (34-kDa translocase of the outer mitochondrial membrane) administered in combination with chemotherapy in patients with metastatic colorectal cancer, a phase I clinical trial with 21 HLA-A2402-positive metastatic colorectal cancer patients was conducted. Patients received a weekly peptide vaccine (1 $\mathrm{mg}$ of each peptide in incomplete Freund's adjuvant) in combination with oral UFT (300 $\mathrm{mg} / \mathrm{m}^{2} /$ day) and UZEL ( $75 \mathrm{mg} /$ day) for 4 weeks, followed by 1 week of rest. The protocol consisted of at least two cycles of this regimen. After the 2nd cycle, vaccinations were given biweekly or monthly, depending on the condition of the patient. Clinical responses were judged 10 weeks after the 2 nd cycle by performing computed tomography (CT) scans and assessing the cytotoxic $\mathrm{T}$ lymphocyte (CTL) responses against RNF43 and TOMM34 in peripheral lymphocytes. The vaccinations were well tolerated without any serious adverse events. CTL responses were induced against both antigens in 8 patients and against one antigen in 12 patients, while 1 patient had no CTL response. The rate of stable disease was $83 \%$. The group with CTL responses against both antigens had the most long-term survivors, followed by the group showing CTL responses against one antigen $(p=0.0079)$. The patients with no CTL responses had the lowest survival. The safety and immunological responsiveness of the present combination therapy suggests that it is clinically beneficial for metastatic colorectal cancer. Further clinical trials are warranted.
\end{abstract}

\section{Introduction}

Genes that are frequently up-regulated in colorectal cancer (CRC) can be identified by genome-wide analysis with cDNA

Correspondence to: Dr Kiyotaka Okuno, Department of Surgery, Kinki University Faculty of Medicine, 377-2 Ohno-higashi, Osakasayama 589-8511, Japan

E-mail: okuno@surg.med.kindai.ac.jp

Key words: peptide vaccine, metastatic colorectal cancer, cytotoxic $\mathrm{T}$ lymphocytes, immunochemotherapy microarray profiling. This strategy has been used to identify gene products that are essential for the proliferation and/or survival of CRC cells (1). Two novel tumor-associated antigens (TAAs), RNF43 (ring finger protein 43) and TOMM34 (34-kDa translocase of the outer mitochondrial membrane), were found to be up-regulated in more than $80 \%$ of CRC tissues as compared to the corresponding noncancerous mucosa $(2,4)$. RNF43 expression cannot be detected in normal human adult organs with Northern blotting. Thus, the function of RNF43 has been associated with the proliferation of tumor cells. Since suppression of TOMM34 by siRNA was found to markedly reduce the growth of colon cancer cells, the gene product is a potential therapeutic target for human CRC (3). HLA-A24-restricted epitope peptides from RNF43 and TOMM34 for cancer vaccination for CRC patients were recently identified $(2,4)$.

We previously reported a phase I trial involving vaccination with cancer peptides in combination with UFT and LV (UZEL) for advanced CRC patients (5). UFT is an oral anticancer drug consisting of tegafur (FT), a prodrug of 5-fluorouracil (5-FU) and uracil, an inhibitor of 5-FU degradation. LV is an oral drug consisting of calcium folinate which modulates 5-FU. We previously demonstrated that the standard dose of UFT and LV did not impede the immunological responses of advanced CRC patients to the peptide vaccination.

To investigate the safety and immunological responses of a peptide vaccination with RNF43 and TOMM34 in combination with UFT and LV, we conducted a phase I clinical study involving patients with metastatic CRC.

\section{Materials and methods}

Patients and eligibility criteria. The study protocol was approved by the Institutional Ethics Review Boards of Kinki University (approval no. 18-15) and was registered in the UMIN Clinical Trials Registry as UMIN000003728 (http://www.umin.ac.jp/ctr/ index.htm). Complete written informed consent was obtained from the patients at the time of enrollment. The patients $(n=23)$ had histologically confirmed metastatic CRC unsuitable for surgical resection and were HLA-A*2402-positive. A total of 19 patients failed to respond to prior standard chemotherapy, and the remaining 4 patients agreed to receive this immunochemotherapy (Table I). Patients were required to have completed prior chemotherapy at least 4 weeks before trial enrollment and to have 
fully recovered from any adverse event with a toxicity of grade 3 or higher according to the Common Terminology Criteria for Adverse Events (CTCAE) scale. The patients were required to have an Eastern Cooperative Oncology Group performance status (PS) of 0 or 1 , to be older than 20 years of age and to have a life expectancy of at least 3 months. Adequate bone marrow (white blood cell count $\geq 3,000 / \mathrm{mm}^{3}$, hemoglobin $\geq 10 \mathrm{~g} / \mathrm{dl}$ and platelet count $\geq 75,000 / \mathrm{mm}^{3}$ ), renal function (serum creatinine $\leq 1.4 \mathrm{mg} / \mathrm{dl}$ ) and liver function (bilirubin $\leq 1.5 \mathrm{mg} / \mathrm{dl}$ and transaminase within 2.5 times the institution's upper limit of normal) were required. Patients were excluded if they were pregnant or had hepatitis $\mathrm{B}$ or $\mathrm{C}$ virus antigens or human immunodeficiency virus (HIV).

Peptides. The RNF43-721 (NSQPVWLCL) and TOMM34-299 (KLRQEVKQNL) peptides were synthesized by American Peptide Company Inc. (Sunnyvale, CA, USA) according to a standard solid-phase synthesis method and purified by reverse-phase high performance liquid chromatography (HPLC) $(4,6)$. The purity $(>95 \%)$ and the identity of the peptides were determined by analytical HPLC and mass spectrometry analysis, respectively. RNF43-721, TOMM34-299 and the epitope peptide derived from the human immunodeficiency virus-envelope (HIV-Env) protein restricted with HLA-A*2402 (RYLRDQQLL) were used to measure the cytotoxic T lymphocyte (CTL) response.

Clinical protocol. The present open-label phase I study involved a vaccine consisting of two peptides $(1 \mathrm{mg}$ of each peptide) derived from RNF43 and TOMM34 mixed with incomplete Freund's adjuvant (IFA) and Montanide ISA 51 (Seppic) administered to patients with locally advanced, recurrent, or metastatic colorectal cancer. The patients received a subcutaneous injection of vaccine into the thigh or back once a week for 5 weeks. Simultaneously, patients received orally administered UFT (300 mg/m²/day) and $\mathrm{UZEL}^{\circledR}$ ( $75 \mathrm{mg} /$ day) for 4 weeks, followed by 1 week of rest (one cycle). The immunological responses to the inoculated peptides and clinical responses were examined after every five vaccinations. The protocol consisted of two cycles. After the second cycle, vaccinations were given biweekly or monthly (depending on patient condition), while UFT/UZEL administration was continued for 4 weeks followed by a 1-week rest period during the entire treatment period. A complete blood count and results of serum chemistry tests were obtained every 2 weeks. Clinical responses were evaluated at the end of every cycle by examining computed tomography (CT) scans and tumor markers. The vaccinated patients $(n=21)$ were assessed for immunological and clinical responses according to the Response Evaluation Criteria in Solid Tumors (RECIST). Signs of toxicity were assessed according to CTCAE version 3.0. Overall survival rates were analyzed by the Kaplan-Meier method, and survival was measured in days from the first vaccination to succumbing to the disease. p-values were assessed using a log-rank test.

Cells. TISI cells and HLA-A*2402-positive B-lymphoblastoid cell lines were purchased from the IHWG Cell and Gene Bank (IHW no. 9042; Seattle, WA, USA) in November 2008 and stored at $-80^{\circ} \mathrm{C}$. Within 2 months of purchase, the cells were resuscitated and maintained in RPMI supplemented with $10 \%$ fetal bovine serum and $1 \%$ penicillin-streptomycin in a humidified $5 \% \mathrm{CO}_{2}$ incubator at $37^{\circ} \mathrm{C}$. The peripheral blood was periodically collected from the enrolled patients. Peripheral blood mononuclear cells (PBMCs) were isolated using FicollPaque Plus (GE Healthcare, Uppsala, Sweden) and density gradient centrifugation and were frozen immediately after isolation. PBMCs from each patient were simultaneously thawed and used to measure the CTL response.

Enzyme-linked immunospot assay. For detecting antigen-specific immune responses, enzyme-linked immunospot (ELISPOT) assays were performed with the human $\gamma$-interferon (IFN- $\gamma$ ) ELISPOT kit (Mabtech, Nacka Strand, Sweden). Plates with 96 wells and nitrocellulose membranes (Millipore, Molshelm, France) were precoated with primary anti-IFN- $\gamma$ antibody (1-D1K) at $4^{\circ} \mathrm{C}$ overnight.

Measurement of the cytotoxic $T$ lymphocyte response. The IFN- $\gamma$ ELISPOT assay was performed to measure the specific CTL response against the peptide. PBMCs were obtained from patients and frozen prior to vaccination and at the end of each treatment course. The frozen PBMCs were thawed and in vitro sensitization was performed. In brief, PBMCs were stimulated with $10 \mu \mathrm{g} / \mathrm{ml}$ of each peptide and $20 \mathrm{IU} / \mathrm{ml}$ of interleukin (IL) -2 at $37^{\circ} \mathrm{C}$, in $5 \% \mathrm{CO}_{2}$ for 2 weeks. Peptides were added on day 0 and 7 . Following incubation, the harvested cells were used as responder cells, and RNF43-721 or TOMM34-299 peptide-pulsed TISI cells were used as stimulator cells $\left(10^{5}\right.$ cells per well). The HLA-A*2402-restricted epitope peptide derived from the HIV-Env protein was used as a control peptide. The IFN- $\gamma$ ELISPOT kit and the AEC substrate set (BD Biosciences Pharmingen, San Diego, CA, USA) were used to measure the CTL response. Spots were captured and analyzed using an automated ELISPOT reader, ImmunoSPOT 4S (CTL Ltd., Cleveland, OH, USA). The ELISPOT assays were performed in triplicate wells. The number of peptide-specific spots was calculated by subtracting the number of spots when stimulated with the HIV-Env peptide from the number of spots when stimulated with the RNF43-721 or TOMM34-299 peptide. The percentage of specific spots was calculated by dividing the number of peptide-specific spots by the number of spots when stimulated with the RNF43-721 or TOMM34-299 peptide. CTL induction was defined as positive when more than 10 specific spots were detected or the percentage of specific spots was greater than $5 \%$. The number of peptide-specific spots was detected as the responder/stimulator ratio-dependency.

Statistical analysis. Overall survival rates were analyzed by the Kaplan-Meier method, and survival was calculated in days from the first vaccination to succumbing to the disease. The statistical analyses were performed with SPSS statistics 17.0 (SPSS, Chicago, IL, USA).

\section{Results}

Characteristics of the patients and vaccinations. Between January 2007 and June 2009, 23 HLA-A*2402-positive patients with metastatic colorectal cancer were enrolled in the present trial. All the patients had one or more metastatic 
Table I. Patient characteristics.

\begin{tabular}{|c|c|c|c|c|c|c|}
\hline $\begin{array}{l}\text { Patient } \\
\text { no. }\end{array}$ & Age & Gender & $\begin{array}{c}\text { Primary } \\
\text { cancer }\end{array}$ & Sites of metastases & PS & Previous treatment \\
\hline 1 & 56 & M & $\mathrm{R}$ & Pelvis & 0 & UFT, CPT-11 \\
\hline 2 & 64 & $\mathrm{~F}$ & $\mathrm{~S}$ & Lung & 0 & 5-FU, UFT/LV \\
\hline 3 & 57 & $\mathrm{~F}$ & $\mathrm{R}$ & Lymph nodes & 1 & 5-FU/LV, CPT-11, S-1 \\
\hline 4 & 42 & M & $\mathrm{R}$ & Pelvis & 0 & None \\
\hline 5 & 53 & $\mathrm{~F}$ & $\mathrm{~S}$ & Lung & 0 & UFT/LV, vaccine \\
\hline 6 & 54 & M & $\mathrm{R}$ & Lung & 0 & None \\
\hline 7 & 74 & $\mathrm{~F}$ & $\mathrm{~S}$ & Lymph nodes & 0 & 5-FU, UFT/LV \\
\hline 8 & 78 & $\mathrm{M}$ & $\mathrm{R}$ & Lung, lymph nodes & 1 & 5-FU, UFT/LV, CPT-11 \\
\hline 9 & 58 & M & $\mathrm{R}$ & Lung & 1 & None \\
\hline 10 & 46 & $\mathrm{M}$ & $\mathrm{T}$ & Liver, lymph nodes & 1 & FOLFOX, FOLFIRI, vaccine \\
\hline 11 & 59 & M & $\mathrm{S}$ & Primary cancer, liver, lymph nodes & 1 & FOLFIRI, FOLFOX \\
\hline 12 & 66 & M & $\mathrm{S}$ & Lung, liver, lymph nodes & 0 & S-1 \\
\hline 13 & 66 & $\mathrm{~F}$ & RS & Lung & 0 & UFT/LV \\
\hline 14 & 49 & M & $\mathrm{S}$ & Lung, liver & 0 & None \\
\hline 15 & 51 & $\mathrm{~F}$ & $\mathrm{~S}$ & Liver, lymph nodes & 1 & UFT/LV, CPT-11 \\
\hline 16 & 66 & M & $\mathrm{R}$ & Lung, liver, lymph nodes & 1 & UFT/LV \\
\hline 17 & 61 & $\mathrm{~F}$ & $\mathrm{C}$ & Liver, lymph nodes & 1 & FOLFOX+Bev, FOLFIRI+Bev \\
\hline 18 & 54 & M & $\mathrm{S}$ & Primary cancer, liver, lymph nodes & 0 & FOLFOX+Bev, UFT/LV \\
\hline 19 & 83 & M & $\mathrm{S}$ & Lung & 0 & UFT \\
\hline 20 & 66 & M & $\mathrm{R}$ & Lung, pelvis, bone & 0 & FOLFOX+Bev, FOLFIRI+Bev \\
\hline 21 & 61 & M & $\mathrm{R}$ & Lung, pelvis & 1 & FOLFOX+Bev, FOLFIRI, CPT-11+Cet \\
\hline 22 & 73 & M & $\mathrm{R}$ & Lung, pelvis, lymph nodes & 0 & FOLFOX+Bev, FOLFIRI, CPT-11+Cet \\
\hline 23 & 65 & M & $\mathrm{R}$ & Lung, pelvis & 0 & FOLFOX+Bev, FOLFIRI+Bev, IRIS \\
\hline
\end{tabular}

PS, Eastern Cooperative Oncology Group performance status; R, rectal cancer; S, sigmoid colon cancer; T, transverse colon cancer; RS, rectosigmoid cancer; C, cecal cancer; Bev, bevacizumab; Cet, cetuximab; IRIS, irinotecan+S-1.

Table II. Adverse events.

\begin{tabular}{lcccc}
\hline Toxicity & $\begin{array}{c}\text { Total } \\
\mathrm{n}(\%)\end{array}$ & Grade 1 & Grade 2 & Grade 3 \\
\hline Anemia & $5(23.8)$ & 5 & 0 & 0 \\
Transaminase elevation & $3(14.3)$ & 3 & 0 & 0 \\
Hyperbilirubinemia & $2(9.5)$ & 2 & 0 & 0 \\
Anorexia & $5(23.8)$ & 2 & 0 & 0 \\
Nausea & $2(9.5)$ & 3 & 0 & 0 \\
Malaise & $3(14.3)$ & 15 & 0 & 0 \\
Vaccination site reaction $^{a}$ & $15(71.4)$ & 0 & 0 & 0 \\
Renal dysfunction $^{\mathrm{a}}$ & $1^{\mathrm{a}}(4.8)$ & 5 & 0
\end{tabular}

${ }^{\text {aA }}$ double-J catheter was placed by a urologist into one patient who experienced acute grade 3 renal dysfunction, which led to the disappearance of the hydronephrosis and the resumption of therapy.

foci that were unsuitable for surgical resection. A total of 19 patients had not responded to prior standard chemotherapy, and the remaining 4 patients agreed to receive this immunochemotherapy (Table I). A total of 2 patients (nos. 10 and 17) were disqualified as they did not meet the inclusion criteria. The final subject group thus consisted of 21 patients $(15$ men and 6 women) with a median age of 61 years (range 42-83). A total of 727 vaccinations were administered with a median of 31 vaccinations per patient (range 7-69). The vaccination with chemotherapy protocol was well tolerated by all patients.

Toxicities. The overall toxicities are shown in Table II. The most frequent adverse events were vaccination-site reactions $(n=15)$, anemia $(n=5)$, anorexia $(n=5)$, malaise $(n=3)$ and 

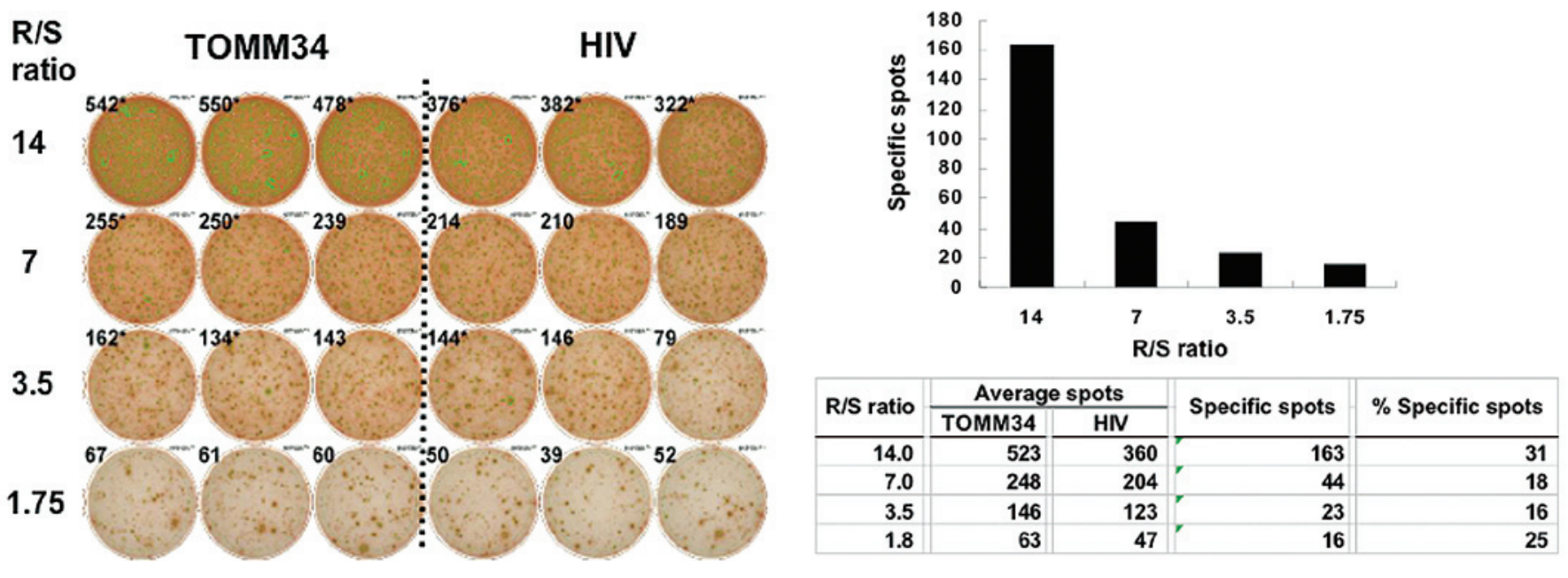

Figure 1. Enzyme-linked immunospot (ELISPOT) assays detecting TOMM34-specific T-cell activity. Peripheral blood lymphocytes collected from patient no. 5 at the end of the second course were cultured in recombinant interleukin-2 without any antigen stimulation for 14 days and subjected to the ELISPOT assay to detect the antigen-specific T-cell response induced by the vaccination.

Table III. Immunological and clinical responses.

\begin{tabular}{rcccccc}
\hline $\begin{array}{l}\text { Patient } \\
\text { no. }\end{array}$ & $\begin{array}{c}\text { No. of } \\
\text { vaccinations }\end{array}$ & $\begin{array}{c}\text { Vaccination } \\
\text { site reaction }\end{array}$ & $\begin{array}{c}\text { CTL } \\
\text { response }\end{array}$ & $\begin{array}{c}\text { Clinical } \\
\text { response }\end{array}$ & TTP (days) & OS (days) \\
\hline 1 & 69 & Ind, red & RNF, TOMM & SD & 252 & 1226 (alive) \\
2 & 7 & $(-)$ & RNF, TOMM & - & 38 & 1026 \\
3 & 17 & Ind, red & TOMM & SD & 169 & 448 \\
4 & 16 & $(-)$ & RNF, TOMM & SD & 211 & 741 \\
5 & 69 & Ind, red & RNF, TOMM & SD & 365 & 1086 (alive) \\
6 & 31 & Ind & RNF, TOMM & SD & 428 & 1054 (alive) \\
7 & 37 & Ind, red & RNF, TOMM & PD & 49 & 1012 \\
8 & 8 & $(-)$ & RNF & - & 36 & 80 \\
9 & 69 & Ind, red & TOMM & SD & 694 & 904 (alive) \\
11 & 11 & $(-)$ & RNF & PD & 36 & 183 \\
12 & 29 & Ind & RNF & SD & 219 & 387 \\
13 & 37 & Ind & TOMM & SD & 219 & 521 \\
14 & 54 & Ind & RNF, TOMM & SD & 260 & 512 (alive) \\
15 & 22 & Ind & RNF & SD & 107 & 197 (alive) \\
16 & 16 & Ind, red & TOMM & SD & 73 & 132 \\
18 & 41 & Ind & TOMM & PD & 70 & 414 (alive) \\
19 & 52 & Ind, red & RNF, TOMM & SD & 309 & 414 (alive) \\
20 & 46 & Ind & RNF & SD & 218 & 330 \\
21 & 50 & Red & TOMM & SD & 246 & 365 (alive) \\
22 & 15 & $(-)$ & TOMM & SD & 69 & 151 \\
23 & 31 & $(-)$ & SD & 176 & 288 \\
\hline
\end{tabular}

Two patients (no.10 and 17) were disqualified for failure to meet the inclusion criteria. CTL, cytotoxic T lymphocyte; TTP, time to progression; OS, overall survival. Ind, induration; red, redness; SD, stable disease; PD, progressive disease.

elevation of serum transaminase $(n=3)$. With the exception of one incident of grade 3 acute renal dysfunction (no. 20) due to hydronephrosis, all of the adverse events were grade 1 . A double-J catheter was placed by a urologist into the patient who experienced acute renal dysfunction, which led to the disappearance of the hydronephrosis and the resumption of therapy. This patient had a large area of tumor recurrence in the pelvis prior to therapy; therefore, the renal dysfunction due to ureteral obstruction was considered to be caused by the metastasis and not related to the therapy.

Immunological monitoring. Peripheral blood lymphocytes obtained before, during, and after the vaccination periods were cultured in rIL-2 without any antigen stimulation for 
A

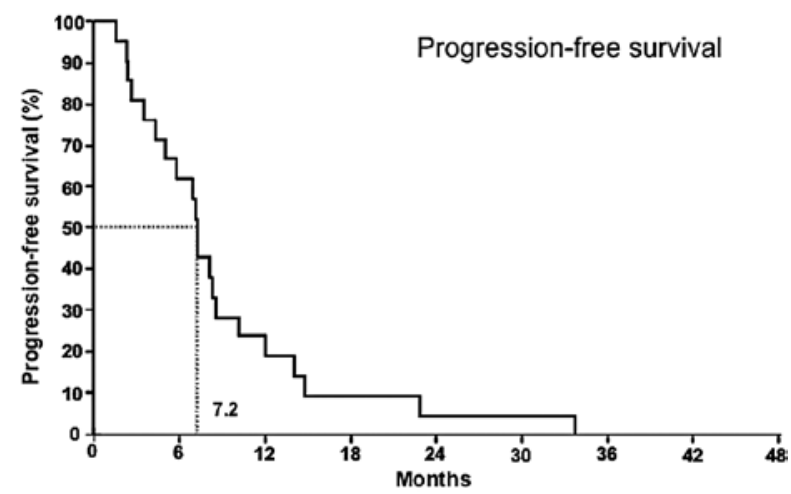

B

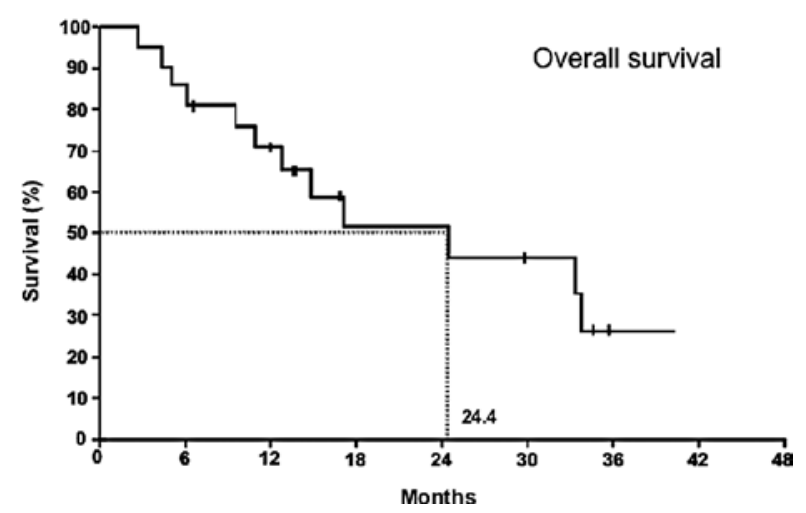

Figure 2. Survival analysis of 21 patients with metastatic colorectal cancer treated with the peptide vaccination in combination with oral chemotherapy. (A) Progression-free survival; (B) overall survival.

14 days and subjected to the ELISPOT assay to detect the antigen-specific T-cell response induced by the vaccination. The CTL response was considered to be positive when more than 10 specific spots were detected or the percentage of specific spots was greater than $5 \%$. In addition, the number of peptide-specific spots was detected as the responder/stimulator ratio-dependency. Representative CTL-positive data from ELISPOT assays against the TOMM34 antigen are shown for patient no. 5 (Fig. 1). Among the 21 patients, 8 patients had positive CTL responses against RNF43 and TOMM34, 12 patients had a positive response against one of the antigens, and the remaining patient had a negative response (Table III). The magnitude of the CTL response varied depending on the timing of the vaccinations. However, there was a clear separation between positive and negative CTL responses.

Clinical response and overall survival. Among the 21 patients, 19 patients were assessed for clinical response at the end of the 10 th vaccination ( 2 nd cycle) according to the RECIST criteria (Table III). The clinical responses of the remaining 2 patients were not assessed as they received fewer than 10 vaccinations (6 and 8, respectively). None of the patients showed a complete response or a partial response. A total of 16 patients had stable disease and 3 patients had progressive disease. The median time of progression-free survival was 7.2 months (Fig. 2A), and the mean survival time was 24.4 months (Fig. 2B).

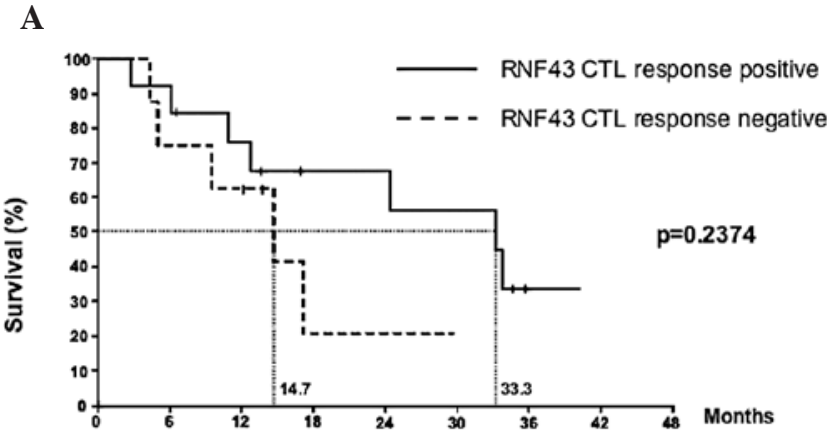

B

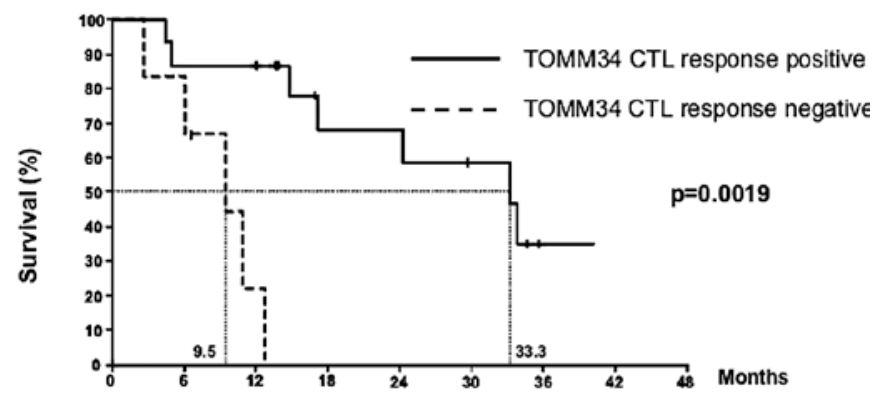

C

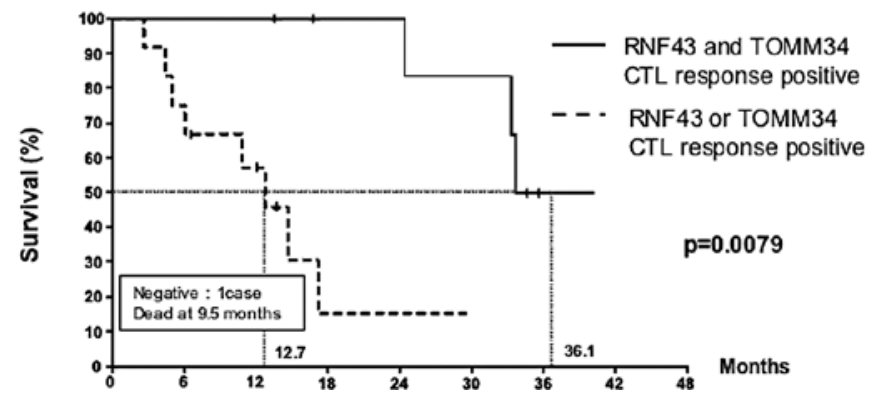

Figure 3. The relationship between cytotoxic T lymphocyte (CTL) responses and overall survival in patients with metastatic colorectal cancer treated with peptide vaccination in combination with oral chemotherapy. (A) CTL response to RNF43 and survival, (B) CTL response to TOMM34 and survival, (C) CTL responses to RNF43 and/or TOMM34 and survival.

Effect of a cytotoxic $T$ lymphocyte response against RNF43 and TOMM34 on overall survival. The effect of a positive CTL response to RNF43 or TOMM34 on overall survival was analyzed. The Kaplan-Meier estimates for the overall survival of patients with detected CTL responses as compared to patients with no response are shown in Fig. 3. No statistical difference was found between the two groups with or without a response to RNF43 ( $p=0.2374$ ) (Fig. 3A). However, there was a statistical difference between the two groups based on the TOMM34 response ( $\mathrm{p}=0.0019)$ (Fig. 3B). Furthermore, we investigated the relationship between CTL response to both antigens and overall survival. The best long-term survival was observed in the group with CTL responses against both antigens, followed by the group showing CTL responses against only RNF43 or TOMM34 ( $\mathrm{p}=0.0079)$. The patient with no response had the lowest survival (Fig. 3C). 


\section{Discussion}

In this clinical trial, cancer vaccination with two peptides in combination with oral UFT/LV chemotherapy was well tolerated without any severe side effects in metastatic CRC patients. Common adverse events included vaccination site reaction, anemia, anorexia, malaise and elevation of transaminase. With the exception of the skin reaction, the rates of other adverse events did not exceed those of the UFT/LV chemotherapy (7). Therefore, addition of the peptide vaccination did not increase the adverse events (beyond mild vaccination site reactions) in this combination therapy. The design of this clinical trial was based on the results of two previous phase I trials. These previous trials found that vaccination with multiple peptides derived from novel cancer-testis antigens in advanced cancer was feasible and that antigen-specific T-cell responses were induced with objective clinical responses (8). These trials also showed that the peptide vaccination combined with oral UFT/LV chemotherapy was well tolerated in the metastatic $\mathrm{CRC}$ patients and induced peptide-specific IgG responses that correlated well with overall survival (5).

The combined chemo-immunotherapy approach has been criticized on the grounds that chemotherapy is immunosuppressive. This opinion is based on the fact that most cytotoxic drugs kill granulocyte precursors in bone marrow and thus induce leucopenia, which is associated with the occurrence of bacterial and mycotic infection. However, there is no evidence that cytotoxic chemotherapy affects the antigen-specific CTL response. Recently, Correale et al (9) reported that the antigen-specific killing ability of human CTL lines in vitro is not affected by 5 -FU or oxaliplatin when exposure to these drugs does not occur during the stimulation phase. Moreover, they found that chemotherapy i) up-regulated tumor-associated antigen expression including CEA or other target molecules such as TS; ii) down-regulated tumor cell resistance to the death signals induced by tumor antigen-specific CTL; iii) reduced the percentage of PBMCs containing immune-suppressive regulatory $\mathrm{T}$ cells $\left(\mathrm{CD} 4{ }^{+} \mathrm{CD} 25^{+} \mathrm{T}\right.$ reg) and the number of cells expressing the FAS receptor (CD95); and iv) induced the complete restoration of the CD4/CD8 T-cell ratio, which is often reduced in advanced cancer patients resulting in a progressively deteriorating immune response (10). Based on these considerations, we believe that the rationale for chemoimmunotherapy in advanced cancer patients will be accepted.

The two cancer-specific peptides, RNF43 and TOMM34, used in the present study are novel cancer-testis antigens specific for CRC. More than $80 \%$ of colorectal cancers express these antigens, and these antigens can induce potent CTLs against colon cancer cell lines $(4,6)$. RNF43 and TOMM34 are defined as oncoantigens. They are highly expressed in cancer cells, are involved in the critical functions of cancer cells (i.e., proliferation) and can induce potent CTL responses. In this context, it is of note that common antigens, such as MUC-1 or CEA, in colorectal cancer, are not critical for tumor cell survival; therefore, they can be lost under the selective pressure of a vaccine-induced antigen-specific immune response without significantly damaging tumor development (11-14).

Using the two crucial cancer-testis antigen-derived peptides, CTL responses were observed in $95 \%$ of the study patients (20 of 21 patients). Potent CTL responses against both antigens were induced in 8 patients (38\%), and a CTL response against one peptide occurred in 12 patients (57\%). Therefore, the use of two peptides allowed CTL responses to occur in almost all patients who received the vaccinations.

Overall survival was well correlated with the response to TOMM34. The patients exhibiting a response to RNF43 also experienced longer survival, although the correlation was not statistically significant. Notably, the patients exhibiting CTL responses to both peptides $(n=8)$ had the longest survival, followed by the patients who showed a CTL response to one peptide $(n=12)$. The patient exhibiting no response had the lowest survival $(n=1)$ (Fig. 2). We do not have evidence to prove that the induced CTLs interacted directly with the cancer lesions in the patients with metastatic CRC to control the cancer lesions and thus contribute to the longer survival. However, we can conclude that the CTL response is a useful biomarker for patients receiving peptide vaccination therapy.

In conclusion, this study suggests that vaccination with two colorectal cancer-specific peptides in combination with $\mathrm{UFT} / \mathrm{LV}$ is well tolerated and can induce potent and specific CTL responses to at least one peptide antigen in $95 \%$ of patients. Furthermore, the patients who developed potent CTL responses against both antigens showed the longest survival. This treatment approach warrants further clinical study.

\section{Acknowledgements}

The authors would like to thank Professor Yusuke Nakamura and Dr Takuya Tsunoda, Laboratory of Molecular Medicine, Human Genome Center, Institute of Medical Science, University of Tokyo, for their excellent advice and cooperation. This study was supported in part by a Grant-in-Aid for Department of the New Energy and Industrial Technology Development Organization (NEDO).

\section{References}

1. Lin YM, Furukawa Y, Tsunoda T, Yue CT, Yang KC and Nakamura Y: Molecular diagnosis of colorectal tumors by expression profiles of 50 genes expressed differentially in adenomas and carcinomas. Oncogene 21: 4120-4128, 2002.

2. Yagyu R, Furukawa Y, Lin YM, Shimokawa T, Yamamura T and Nakamura Y: A novel oncoprotein RNF43 functions in autocrine manner in colorectal cancer. Int J Oncol 25: 1343-1348, 2004.

3. Chewawiwat N, Yano M, Terada M, Hoogenraad NJ and Mori M: Characterization of the novel mitochondrial protein import component, Tom34, in mammalian cells. J Biochem 125: 721-727, 1999.

4. Shimokawa T, Matsushima S, Tsunoda T, Tahara H, Nakamura Y and Furukawa Y: Identification of TOMM34, which shows elevated expression in the majority of human colon cancers, as a novel drug target. Int J Oncol 29: 381-386, 2006.

5. Hattori T, Mine T, Komatsu N, et al: Immunological evaluation of personalized peptide vaccination in combination with UFT and UZEL for metastatic colorectal carcinoma patients. Cancer Immunol Immunother 58: 1845-1854, 2009.

6. Uchida N, Tsunoda T, Wada S, Furukawa Y, Nakamura Y and Tahara H: Ring finger protein 43 as a new target for cancer immunotherapy. Clin Cancer Res 10: 8577-8586, 2004.

7. Shirao K, Hoff PM, Ohtsu A, et al: Comparison of the efficacy, toxicity, and pharmacokinetics of a uracil/tegafur (UFT) plus oral leucovorin (LV) regimen between Japanese and American patients with advanced colorectal cancer: joint United States and Japan study of UFT/LV. J Clin Oncol 22: 3466-3474, 2004.

8. Kono K, Mizukami Y, Daigo Y, et al: Vaccination with multiple peptides derived from novel cancer-testis antigens can induce specific T-cell responses and clinical responses in advanced esophageal cancer. Cancer Sci 100: 1502-1509, 2009. 
9. Correale P, Del Vecchio MT, Genova GD, et al: 5-Fluorouracilbased chemotherapy enhances the antitumor activity of a thymidylate synthase-directed polyepitopic peptide vaccine. J Natl Cancer Inst 97: 1437-1445, 2005.

10. Correale P, Cusi MG, Tsang KY, et al: Chemo-immunotherapy of metastatic colorectal carcinoma with gemcitabine plus FOLFOX 4 followed by subcutaneous granulocyte macrophage colony-stimulating factor and interleukin-2 induces strong immunologic and antitumor activity in metastatic colon cancer patients. J Clin Oncol 23: 8950-8958, 2005.

11. Nagorsen D and Thiel E: HLA typing demands for peptide-based anti-cancer vaccine. Cancer Immunol Immunother 57: 1903-1910, 2008.
12. Liu K, Wang C, Chen L, et al: Generation of carcinoembryonic antigen (CEA)-specific T-cell responses in HLA-A0201 and HLA-A2402 late-stage colorectal cancer patients after vaccination with dendritic cells loaded with CEA peptides. Clin Cancer Res 10: 2645-2651, 2004.

13. Weihrauch MR, Ansen S, Jurkiewicz E et al: Phase I/II combined chemoimmunotherapy with carcinoembryonic antigen-derived HLA-A2-restricted CAP-1 peptide and irinotecan, 5-fluorouracil, and leucovorin in patients with primary metastatic colorectal cancer. Clin Cancer Res 11: 5993-6001, 2005.

14. Dittmann J, Keller-Matschke K, Weinschenk T et al: CD8 ${ }^{+} \mathrm{T}$-cell response against MUC-1-derived peptides in gastrointestinal cancer survivors. Cancer Immunol Immunother 54: 750-758, 2005. 